Cinémas

Revue d'études cinématographiques

Journal of Film Studies

\title{
L'infatigable image ou les horizons du temps au cinéma
}

\section{Lucie Roy}

Volume 5, numéro 1-2, automne 1994

Le Temps au cinéma

URI : https://id.erudit.org/iderudit/1001011ar

DOI : https://doi.org/10.7202/1001011ar

Aller au sommaire du numéro

Éditeur(s)

Cinémas

ISSN

1181-6945 (imprimé)

1705-6500 (numérique)

Découvrir la revue

Citer cet article

Roy, L. (1994). L’infatigable image ou les horizons du temps au cinéma.

Cinémas, 5(1-2), 147-166. https://doi.org/10.7202/1001011ar
Résumé de l'article

L'auteure propose de réfléchir sur le statut photographique du film et, plus précisément, sur les enjeux d'une " conscience d'image " ou d'une intentionnalité inscrite dans le film, laquelle peut faire jouer la « rétention avec perception " et le " ressouvenir sans perception » directe (Ricoeur). L'auteure propose de lire le film comme une série de perceptions qui résulte d'une oeuvre cognitive et a pour origine une pensée en écriture et, du côté spectatoriel, de le considérer comme une oeuvre perceptive, une oeuvre offerte à la perception et dont la compréhension dépend du recouvrement cognitif effectué pat le spectateur. Aux deux pôles, de la perception par le spectateur de l'écriture cognitive du film et de la cognition en forme de perception dans le film, le film trace le réseau d'une phénoménalité. 


\title{
L'infatigable ${ }^{1}$ image ou les horizons du temps au cinéma
}

\section{Lucie Roy}

\begin{abstract}
RÉSUMÉ
Lauteure propose de réfléchir sur le statut photographique du film et, plus précisément, sur les enjeux d'une "conscience d'image" ou d'une intentionnalité inscrite dans le film, laquelle peut faire jouer la "rétention avec perception" et le "ressouvenir sans perception" directe (Ricœur). Lauteure propose de lire le film comme une série de perceptions qui résulte d'une œuvre cognitive et a pour origine une pensée en écriture et, du côté spectatoriel, de le considérer comme une œuvre perceptive, une œuvre offerte à la perception et dont la compréhension dépend du recouvrement cognitif effectué par le spectateur. Aux deux pôles, de la perception par le spectateur de l'écriture cognitive du film et de la cognition en forme de perception dans le film, le film trace le réseau d'une phénoménalité.
\end{abstract}

\section{ABSTRACT}

In this article the author reflects on film's status as photography, or more specifically on what is at stake in an "image-consciousness," or an intentionality in the making of a film. This brings into play the direct "retention with perception" and the "re-remembering without perception" (Ricœur). Moreover the author proposes reading film as a series of perceptions which result from cognitive work and originate from a thought in writing and, on the spectatorial side, as perceptive work, one offered to the perception, whose understanding depends on the cognitive retrieval carried out by the spectator. Between the two poles, from the perception by the spectator of the cognitive writing 
of the film and the cognition of the form of perception in the film, the film traces a network of phenomenality.

Je trouvai d'abord ceci. Ce que la Photographie reproduit à l'infini n'a eu lieu qu'une fois: elle répète mécaniquement ce qui ne pourra jamais plus se répéter existentiellement. En elle, l'événement ne se dépasse jamais vers autre chose: elle ramène toujours le corpus dont j'ai besoin au corps que je vois; elle est le Particulier absolu, la Contingence souveraine, mate et comme bête, le Tel (telle photo, et non la Photo), bref, la Tuché, l'Occasion, la Rencontre, le Réel, dans son expression infatigable.

Roland Barthes, La Chambre claire

Empruntant, pour les prolonger, certains aspects théoriques proposés par Roland Barthes dans La Chambre claire, qui s'est attaché à comprendre l'émergence du sens dans l'image photographique, je me penche sur l'étude du statut spectral, le "spectrum ", de l'image cinématographique et de son double, le "punctum", en forme notamment de recouvrement par le spectateur.

Or, je le souligne, il ne s'agit pas de suivre simplement ici le jeu énonciatif du film qui, de son point d'origine jusqu'à son point d'arrivée, de son énonciation à sa réception, fait lire en dessinant et fait comprendre en en permettant la lecture, mais bien de mieux saisir la complexité relative de son noyau en lui restituant son épaisseur temporelle et, pour en initier les termes, les horizons de temps auxquels correspondent des couches mémorielles. Cet objectif d'enquête implique non seulement l'incontournable examen taxinomique "des images et des signes" (Deleuze, 1983), mais il induit tout d'abord, et j'oserais dire davantage, l'étude de l'écriture cinématographique ou, pour être plus précise, celle de la cristallisation ${ }^{2}$ des temps par le récit. 
Dans cette perspective, je m'attache en première partie de l'analyse, "Le cinéma ou le Tel infatigable de l'image: le punctum temporel ", à vérifier, pour employer autrement l'expression de Roland Barthes, le degré d' "adhérence référentielle " ${ }^{3}$ " $d u$ temps à l'image, celui donc de la découpe et de la prise des temps, celui des coupes temporelles successives en forme de syntaxe par laquelle je considère la coprésence des sons et des images et celui, enfin, en quelque sorte exposant des deux premiers états, du réseau des temps de monde inscrits dans le récit. Dans la seconde partie, "Le cinéma ou le punctum mémoriel ", j'aborde la question cognitive des tracés de la mémoire appelés par l'écriture filmique se faisant récit. Enfin, en troisième et dernière partie, "La conscience d'image", je remets au jeu les questions d'intentionnalité et de temporalité dans l'image du film.

J'interroge également, mais par examen de son contraire, l'énoncé de Roland Barthes qui a trait à l'«indissociabilité de la vitre et du paysage ${ }^{4}$ " de la photographie et j'avance, en ce qui concerne l'écriture filmique, la prémisse d'une non-transparence de la vitre et du paysage ou, excluant cette dernière métaphore, celle du cadre et de l'espace représenté. J'explore, en somme, les apparents "non-temps" ": ceux qui se dessinent hors du champ de la perception écranique et ceux qui, en forme d'ellipses et de résurgences temporelles, participent de la configuration du récit.

Bref, je tente de comprendre comment le film, du matériau au récit, dessine des "zébrures ${ }^{6}$ " de temps et, comme il en est de sédiments déposés sur la surface terrestre, des horizons ${ }^{7}$ temporels. L'image est, en somme, dans son épaisseur autant que dans sa traversée, vue comme possible, comme un réseau d'arborisations qui tantôt - je cite presque Ropars - fait écran en jouant la vue et tantôt écranise en voilant, alors que, grâce à elles — l'image, son épaisseur et sa traversée —, le récit et les mondes possibles qui y sont compris prennent part à une logique temporelle. Le film met en présence des formes temporelles par l'appel du temps dans le et, davantage, hors du site du film.

En fait, il est ici question du "Tel" infatigable de l'image, mais d'un " Tel" aux prises avec le continuel battement de la discontinuité filmique, un "Tel" sans même parce que celui-ci est soit fictionnalisé, soit documentarisé, un "Tel " enchâssé dans la 
voûte de l'écrire, un "Tel " détourné, différencié et recouvert: un "Tel », mais mis au jeu du mémoriel et de l'« immémoriel».

\section{Le cinéma ou le Tel infatigable de l'image : \\ le punctum temporel}

Parce que cette enquête ne s'attache pas, comme on vient de le voir, spécifiquement à l'étude de la photographie, je me permets d'approfondir dans une autre perspective la dyade ou, mieux, l'accolade "spectrum/punctum " énoncée par Roland Barthes.

Le "spectrum" comprend, selon Barthes, "[...] celui ou cela qui est photographié, [...] la cible, le référent, sorte de petit simulacre $[\ldots]^{8}$, parce que ce mot garde à travers sa racine un rapport au spectacle" (p. 22-23). Le terme "punctum" désigne, quant à lui, "[...] non plus uniquement [...] cette blessure, cette piqûre, cette marque faite par un instrument pointu; [cette] idée de ponctuation [...]" (p. 49); ce qu'il désigne aussi "[...] ce nouveau "punctum", qui n'est plus de forme, mais d'intensité, c'est le Temps, c'est l'emphase déchirante du noème (ça-a-été), sa représentation pure" (p. 148).

Aux deux aspects du "punctum" définis par Roland Barthes pourrait s'ajouter pour le cinéma un troisième effet de ponctuation ou de temporalisation. Ce "punctum " ainsi considéré comprendrait non seulement le "ce-qui-à-l'image-me-point ${ }^{9}$ " et la brisure du temps qui retient le passé, mais il inclurait aussi la syntaxe du film qui, en termes de simultanéité et de continuité de simultanéités, fait basculer ou muter les perpétuelles fêlures temporelles de l'image en "énoncé métaphorique ${ }^{10}$ " de temps, en récit donc.

De quelques façons qu'on le prenne, le cinéma ne fait pas, comme en photographie, seulement jaillir les objets, les visages et les paysages, en en jouant le simulacre et en retenant un certain "ça-a-été", mais il énonce un enchevêtrement d' étales " ", voix et images qui constituent des manifestes de temps. Parce qu'il est ici question du matériau et des formes temporelles qu'il emprunte, cette étude ne concerne donc pas uniquement l'image filmique, mais elle se préoccupe aussi de l'examen de la voix en tant qu'elle agit et peut agir en rapport notamment avec 
l'image, comme empreinte et spectre de temps autre au cinéma ${ }^{12}$, de sorte que, dans l'intervalle des sons et des images, comme dans les sons, les images et les intervalles séquentiels eux-mêmes, peut se tisser et se tisse effectivement un réseau temporel ${ }^{13}$.

$\mathrm{Si}$, par suite et aux termes de mon analyse, cette entreprise veut considérer le mouvement des arrêtés et des avancées du temps dans la syntaxe filmique, il importe d'examiner, à l'instar de Paul Ricœur pour la littérature, le champ d'exploration plus que d'application - au cinéma du principe de "rétention" et de "protention" tel que défini par Husserl. Pour en marquer l'usage, on pourrait dire de façon provisoire que la rétention participe, par intentionnalité, de la retenue du passé dans le présent, alors que la protention anticipe l'avancée du temps dans ce même "alentour temporel du présent" (Ricœur, 1985, p. 70). Reste à définir pour le cinéma à quoi correspond ce "ce" qui joue de la retenue ou de l'anticipation du temps.

Une double précision s'impose à ce propos. La première veut que ce principe de rétention et de protention soit dans l'écriture filmique, en simultanéité comme en continuité, fondateur de l'épaisseur et des horizons du temps et que celui-ci s'inscrive dans le "comme-si " de la représentation: "Il en résulte que tout moment de la suite des instants présents [dans le film] peut être re-présenté [...] comme présent-source sur le mode du "comme-si". Ce quasi-présent-source aura donc son halo temporel, qui en fera chaque fois le centre de perspective pour ses propres rétentions et protentions" (Ricœur, 1985, p. 63). La deuxième précision veut que soit préalable à cette épaisseur et à ces horizons du temps l'existence d'une "conscience-temps" (p. 44-45) ou, si l'on veut, celle d'une pensée en écriture prise dans l'écranicité temporelle du circuit filmique. En ce sens, du "punctum " ou du "ce " " qui-à-l'image-me-point " dépendrait la saisie par le spectateur de cette pensée en écriture ${ }^{14}$ stipulée ou ponctuée dans et par la phénoménalité de l'écriture photographique ou filmique. "Toute conscience, disait Husserl, se caractérise relativement à un objet "(Husserl, 1970a, p. 21) et, j'ajoute, toute pensée, même en écriture ou en lecture spectatorielle, se caractérise relativement au temps de l'énonciation et au temps toujours pluriel de la pensée qui énonce et comprend. 
Le jaillissement pour ainsi dire noématique de l'image relève, pour la phénoménologie, d'une intentionnalité et que son adresse, comme sa compréhension par le spectateur, participe à son tour d'un recouvrement ou, en d'autres termes, de la lecture - intentionnelle - d'une intentionnalité comme autre qui, en forme d'empreinte, demeure dans le gel mouvant de l'écriture. Or, cette écriture-ci est vue "[...] dans sa plénitude, c'est-à-dire comme corrélat ou "produit " intentionnel [qui vient], non d'une perception isolée mais du "système » infini des vécus cognitifs qui sont précisément constitutifs du monde comme existant " (Husserl, 1970b, p. 14).

Dans cette perspective, et ne serait-ce que parce que le film se nourrit de façon écranique de l'apparaître du temps et de l'espace, l'on pourrait croire qu'étant intentionnel, le film convoque d'ores et déjà l'infini des vécus cognitifs. S'inspirant du projet phénoménologique husserlien, on serait enclin à croire que: " [...] si le procès filmique donne à contempler des apparences en se faisant apparence de monde, $s^{\prime}$ il laisse se profiler des silhouettes ou des ombres de monde, c'est que détournant la réalité [de son cours], il donne à lire une émotion de monde, [une visée], un certain type d'intentionnalité, une perception cognitive [...]" (Roy, 1988-89, p. 104). Bref, le film participe d'un état transitif et contient, par arrêtés et, davantage, par appels de la signification, le tout des vécus cognitifs.

Cela dit à titre de parenthèse, je ne pose pas pour des équivalents sémiotiques le mot et l'image à l'écran, mais considère le tissu de leur phénoménalité langagière, laquelle participe, selon Husserl, d'une noèse, - d'un acte de connaissance, d'une certaine intentionnalité donc - et d'un noème - d'une connaissance en tant que résultat ${ }^{15}$. Je considère, enfin, dans le film et plus largement encore dans le cinéma, l'empreinte en même temps que la traversée ou, pour en quelque sorte réciproquement poser leurs homonymes, la rétention et la protention, en tant qu'œuvres de la pensée dans la course filmique.

J'aimerais tenter de clarifier autrement les quelques inspirations théoriques qui fondent l'actuel projet d'analyse en traçant, pour les définir, les contours des horizons temporels au cinéma qui, je le souligne, courent sur toute la surface du film, du maté- 
riau à sa syntaxe, du film à son identité cognitive, bref, de son écriture à sa réception.

On serait, dans la perspective ici engagée, enclin à supposer :

i) que chaque état spectral d'image réfere à la brisure d'un continuum temporel et implique, ce faisant, par recouvrement, un certain "punctum" temporel, de même qu'une épaisseur temporelle qui comprend les apparents non-temps;

ii) que l'image filmique peut participer d'un état spectral, par endroits, superficiellement assez semblable à celui de l'image photographique qui laisse, selon Barthes, resurgir un certain "ça-a-été". Je dis, "par endroits", dans la mesure où il faudrait approfondir les règles phénoménologiques du temps en ce qu'il "apparaît " à l'image du film et, sur le plan de la narrativité cette fois-ci, réfléchir à une logique temporelle des mondes possibles. Par rapport à ces règles phénoménologiques du temps et à cette logique temporelle, la lecture de ce "ça-a-été" serait, il va sans dire, plus complexe. On le voit, la question ici posée concerne, en bout de course, les sites de référence et d'appartenance des mondes qui ont à la fois prise sur du temps "réel " et du temps "en " récit.

iii) que l'appareil cinématographique tisse, au regard de l'a priori de ce "ça-a-été", le réseau de ses différences. On parlerait ainsi d'un "ça-a-été" relatif, d'un "ça-a-été» de l'ordre de l'écriture et on pourrait aussi faire référence au statut d'existence du film correspondant au sillon de l'intentionnalité qui, aux deux extrêmes, serait de type documentarisant ou fictionnalisant ;

iv) que chacun des états temporels du film se trouve traversé, hiérarchisé et substantialisé par l'identité d'un certain vécu cognitif ${ }^{16}$. Cette hiérarchisation des formes temporelles par laquelle un temps premier retient un autre temps - ou un temps autre - est possible grâce à l'existence d'une certaine épaisseur temporelle dont la compréhension découle de la somme des vécus cognitifs. De sorte qu'au cinéma, l'actualité du temps de la perception permettrait, du côté de la réception, la remontée 
d'un autre temps qui, contemporain dans et par la manifestation de l'écriture filmique, peut phénoménalement raviver un certain "ça-a-été", un temps passé ou lointain;

v) que cette traversée de la pensée à l'image, s'accordant si l'on veut à la prise et à l'emprise d'un certain vécu cognitif dans le film, agirait constamment comme un "punctum", un "ce-qui-à-l'image-me-point" ou me saisit, comme une intentionnalité, même lorsque neutre ou neutralisée par son apparente absence à l'écran ;

vi) que l'émergence du sens temporel à l'image filmique dépendrait de la contraction du rétentionnel — dans son épaisseur - et du protentionnel - dans la succession des épaisseurs temporelles - sous le couvert de cette même intentionnalité qui en guide l'écriture et, autrement, la lecture ;

vii) que le film réfere, par son écriture, à l'identité et à la somme des vécus cognitifs en jouant et en recouvrant l'«immémoriel» (fictionnel) et / ou le mémoriel (factuel) ou en jouant - ce qu'il fait toujours - de l'opération de recouvrement de l'«immémoriel " et/ou du mémoriel par le fictionnel.

L'analyse ne recourt, comme on le voit, ni à la sémiologie, ni à la narratologie, ni à certaines des théories plus anciennes de la communication à partir desquelles on a par ailleurs défini mais autrement, au sortir de la boîte à message ou au sortir du film, le texte comme relevant d'une intentionnalité d'émission. Elle s'attache à l'étude de la phénoménalité du film et s'applique conséquemment à l'étude des horizons temporels tels que précédemment envisagés, plutôt que définis. Elle se préoccupe aux termes de l'analyse de l'examen du relatif étalement des temps par étagement des formes parfois estompées du temps au cinéma. 


\section{Le cinéma ou le "punctum» mémoriel}

Toute photographie est un certificat de présence.

Roland Barthes, La Chambre claire

Je m'applique à décrire le tout premier segment du film Shoah de Lanzmann (1985). Cette séquence, particulièrement exemplaire quant aux méthodes narratives empruntées, me sert de lieu de démonstration.

Des écrits défilent à l'image:

Laction commence de nos jours à Chelmno sur Ner, Pologne. À 80 kilomètres au nord-ouest de Lodz, au cœur d'une région autrefois à fort peuplement juif, Chelmno fut en Pologne le site de la première extermination de Juifs par le gaz. [...]. Sur les 400000 hommes, femmes et enfants qui parvinrent en ce lieu, on compte deux rescapés: Mordechaï Podochlebnik et Simon Srebnik. Simon Srebnik, survivant de la dernière période, était alors un enfant de treize ans et demi. Son père avait été abattu sous ses yeux, au ghetto de Lodz, sa mère asphyxiée dans les camions de Chelmno. Les SS l'enrôlèrent dans un des commandos de "Juifs du travail ", qui assuraient la maintenance des camps d'extermination et étaient eux-mêmes promis à la mort. [...].

Dans la nuit du 18 janvier 1945, deux jours avant l'arrivée des troupes soviétiques, les nazis tuèrent d'une balle dans la nuque les derniers "Juifs du travail". Simon Srebnik fut exécuté lui aussi. La balle ne toucha pas les centres vitaux. Revenu à lui, il rampa jusqu'à une soue à cochons. Un paysan polonais le recueillit. Un médecin-major de l'Armée rouge le soigna, le sauva. [...]. C'est en Israël que je l'ai découvert. J'ai convaincu l'enfant chanteur de revenir avec moi à Chelmno. Il avait 47 ans $^{17}$.

Simon, immobile, dans une embarcation poussée par un canotier, file doucement sur un cours d'eau peu profond. Après 
un long silence, il chante a capella. La caméra les suit. En voix off, on traduit: "Il avait treize ans et demi, il avait une très belle voix. Il chantait d'une façon très belle et on l'entendait. " Simon chante toujours. En voix off, une femme raconte: "Quand je l'ai entendu, mon cœur a battu beaucoup plus fort, parce que ce qui s'est passé ici, c'était un meurtre. J'ai vraiment revécu ce qui s'est passé." Simon, silencieux, marche sur une route. "Difficile à reconnaître, dit-il — et lit-on en sous-titres —, c'était ici. Ici, on brûlait les gens. Beaucoup de gens ont été brûlés ici. Oui, c'était le lieu ". L'image, par un panoramique, donne à voir un champ où l'on peut reconnaître les fondations d'anciens bâtiments. "Personne, poursuit-il, n'en repartait jamais. " La caméra revient sur Simon et le cadre en plan poitrine. "Les camions à gaz arrivaient là... Il y avait deux immenses fours... et ensuite, on jetait les corps... dans ces fours, et les flammes montaient jusqu'au ciel. - Jusqu'au ciel ?" interroge Lanzmann. "Oui. C'était terrible", répond Simon. Il marche dans le même champ, la caméra le suit. "On ne peut pas raconter cela. Personne ne peut... se représenter ce qui s'est passé ici. Impossible. Et personne ne peut comprendre cela. Et moi-même aujourd'hui..." Lanzmann marche à la même hauteur que Simon. Puis, seul, Simon marche sur la longue dalle d'un des bâtiments. "Je ne crois pas que je suis ici. Non, cela, je ne peux pas le croire. C'est toujours aussi tranquille ici, toujours. Quand on brûlait chaque jour deux mille personnes, des Juifs, c'était également tranquille. Personne ne criait, chacun faisait son travail. C'était silencieux, paisible. Comme maintenant."

Dans le segment filmique précédemment décrit, on a affaire à une double prise temporelle et à la mise au jeu du pouvoir d'authentification temporelle de l'image par le détournement de son habituelle force "constative" ou figurative ${ }^{18}$. La première saisie temporelle correspondrait à l'apparition du temps écranique ou à la configuration par l'image d'un certain "ça-a-été", manifestation de l'ancienne capture du temps par la mécanique cinématographique. La seconde saisie, en transparence dans l'apparence figurative et temporelle de la première, emporterait un autre temps, mais plus ancien, celui de Chelmno, 1942, que se remémore Simon et que s'attache à rappeler Lanzmann. 
Le film use d'images "mélancoliques ${ }^{19}$ " pour employer une fois encore la terminologie de Roland Barthes, parce que restées engorgées dans un hors-temps, parce que dénuées du plein pouvoir constatif de l'apparition ou de la re-présentation ${ }^{20}$. Mélancoliques, parce qu'elles font surgir, comme par derrière, une perception temporelle, mais médiatisée par la première et notamment appelée par la voix, celle, principalement, de Simon.

Vu dans cette perspective, l'espace écranique rappellerait, par sa contemporanéité même, cette persistance du temps, l'implication d'un "ça-a-été" dans l'actualité perceptive, rétentionnelle, que donnent à voir les images du film et l'implicitation d'un "çaa-été" plus lointain encore, un "ça-a-été" de l'histoire que donnent, cette fois-ci, à comprendre les voix et les mentions écrites.

Image-relais, cette séquence filmique recèle, en d'autres termes, une certaine "passéité" dans le tracé d'une contemporanéité en quelque sorte lestée par la pensée de ses frontières temporelles. Il appert, en somme, qu'ici plus qu'ailleurs l'« [...] analyse du temps [...] est d'emblée une analyse de la durée, au sens de la continuation, de la persistance considérée comme telle [...], et non pas seulement de la succession " (Ricœur, 1985, p. 51). Il semble aussi que les deux forces rétentionnelles du récit, la coïncidence et, j'oserais même dire, la disjonction temporelle du "quasi-présent" (p. 62) retenu par l'image et la voix pour un passé par leur entremise convoqué, et celle aussi de l'image — qui retient en trace $d u$ temps passé - par rapport à la voix - qui rappelle le temps passé ou en quelque sorte l'«identité" de ce temps passé -, participent à l'intérieur des moments du film de l'insistance impressionnelle ${ }^{21}$ du sens (et) de la temporalité dans le film, de sorte que, dans la découpe des voix et des images filmiques, dans les bords vifs des coupes de temps par elles fabriquées peuvent se distinguer, au cinéma, des "images-perception" de même que des images dont les perceptions débordent largement sur le plan du sens celles déjà comprises dans la course du récit filmique : là où le temps hors-récit persiste et dure par recouvrement et remémoration.

$\mathrm{Si}$, aux termes d'une phénoménologie de la réalité se distinguent " [...] la rétention qui se constitue en continuité avec la 
perception et le ressouvenir qui seul est, au sens fort du mot, une non-perception" (Ricœur, 1985, p. 55), l'on peut, mais de façon différente parce que le cinéma joue, on l'a dit, par avance le manifeste de la perception par la mise en temps des lieux de la réalité, prétendre que le film peut pratiquer la rétention avec visée perceptive et, dans l'intervalle de ces mises en temps des lieux de la réalité, comme dans l'intervalle creusé entre les voix et les images, le ressouvenir sans visée perceptive directe.

J'ajoute, à titre de parenthèse, que j'aurais pu autrement et uniquement m'occuper de la seule analyse des paroles et de leur figuration remémorante, mais ce faisant je n'aurais pas été fidèle à l'intrication visuelle et sonore du film. Pour être encore plus précise, ces images dont il est ici question sont d'ordre photographique/ filmique, mais elles sont aussi d'ordre langagier, de l'ordre donc, de l'effectuation de la pensée par des images visuelles et sonores. Ainsi il est vrai de dire qu'il y a, dans le film Shoah, du ressouvenir sans visée perceptive directe, sans figuration directe donc par l'image des événements qui ont eu cours dans le temps passé. Mais il est encore plus vrai de dire que ce ressouvenir sans visée perceptive directe est dû à l'apparaître d'une intentionnalité en forme - par les images et les voix de faire-absence ou d'un faire qui présente l'absence.

Faisant appel à la voix pour raviver le passé historique, laissant ainsi hors jeu la perception directe du temps que des images des camps d'extermination auraient pu reconduire dans l'appareillage figuratif du film et rappelant conséquemment le passé moins lointain de la prise écranique, le film Shoah semble se prêter à la fois au jeu de la rétention et à celui du ressouvenir. Le "ça-a-été " de la prise écranique marque ici effectivement le passage et l'antériorité d'un autre temps dans le présent ou le "quasi-présent" écranique, en forme d'irreprésentable d'abord, de ressouvenir ensuite. "Le ressouvenir [même par le film reproduit] n'est [donc] pas seulement un "comme-si " présent: il vise le présent et ainsi le pose comme ayant-été " (Ricœur, 1985, p. 68), une intentionnalité, une volonté discursive ressouvenante. En sorte que du "quasi-présent" du film ressort l'idéation du temps passé, son devenir pour une part intentionnellement présent - c'est le principe de la rétention et de l'horizon 
d'attente qu'il dessine - et, pour une autre part, sur le plan de la figuration des événements, intentionnellement absent - sans avenir - parce que sans perception directe.

L'on peut par là concevoir que, de façon générale, même plongées dans les silences, même détournées de leur usuel pouvoir d'authentification, la voix et, davantage, l'image peuvent faire poindre l'horizon d'un autre temps comme tel in-manifestable, parce que notamment pris dans la chute du passé. Et il peut configurer par intentionnalité l'infigurable du temps passé par, justement, l'absence de sa perception directe par l'image. Ce temps-ci s'écrit par imprésence de l'écrire qui appelle inlassablement le recouvrement du récit par la mémoire et les horizons du temps qu'elle traverse et qu'elle a traversés. L'écranicité fait ici, dans le film Shoah, coïncider l'in-manifestable du temps avec la figuration - relativement infigurante - d'une intentionnalité temporelle remémorante ${ }^{22}$.

En somme, on pourrait dire que, si le film donne à apparaître des temps en forme de rétention et de ressouvenir, s'il conjugue, sous le joug d'une certaine phénoménalité, des temps de mondes dans le et hors du site du film, si enfin le film par ses interstices compris entre les voix et les images, et dans les "images-temps" elles-mêmes, dessine des zébrures et des horizons temporels, c'est que, s'adressant au spectateur, il l'enjoint de participer par recouvrement à l'œuvre de remémoration du film sans laquelle la forme filmique resterait comme "mate " et "bête".

"Conscience d'image ${ }^{23}$ "

Nous n'insérons le vécu dans aucune réalité. Nous n'avons affaire à la réalité que dans la mesure où elle est visée, représentée, intuitionnée, conceptuellement pensée.

Husserl

Mais j'aimerais approfondir l'idée de Barthes qui veut que: "Dans cette recherche de la Photographie [et, pour ce qui concerne le présent travail, de la cinématographie], la phénoméno- 
logie [prête] un peu [et même beaucoup] de sa langue" (p. 40) et montrer, sous forme d'essai, que le cinéma ressemble à la langue d'une phénoménalité. On pourrait, en effet, vouloir mieux délimiter les frontières de la discipline philosophique qu'est le projet phénoménologique qui, il est vrai, concerne la description ${ }^{24}$ des phénomènes, même langagiers, et celle d'une écriture de la phénoménalité qui appelle et décrit des phénomènes, mais tirés du champ phénoménal de l'expérience humaine: le temps, la mémoire, l'imaginaire, la quotidienneté, etc.

Dans la perspective donc du dessin de la phénoménalité d'autres diraient du projet phénoménologique - du film qui trace les traits de la connaissance par figuration et représentation est rapportée l'idée d'une intentionnalité filmique et temporelle que le projet phénoménologique husserlien peut contribuer à éclairer encore davantage. Une précision s'impose par avance: le film offre, en séries, des perceptions qui résultent d'une œuvre cognitive et qui ont pour départ une pensée en écriture; du côté spectatoriel, il offre une œuvre perceptive, une œuvre offerte à la perception et dont la compréhension dépend du recouvrement cognitif effectué par le spectateur. Aux deux pôles, celui de la perception par le spectateur de l'écriture cognitive du film et celui de la cognition en forme de perception dans le film, le film trace le réseau d'une phénoménalité.

Lintentionnalité filmique correspond ou pourrait correspondre, pour une part, à l'activité de la conscience ou, mieux, à celle de la pensée en écriture, à son apparaître et à son faire apparaittre. Celle-ci déployant filmiquement des phénomènes, et retournant, on l'a dit, aux phénomènes eux-mêmes, énonce en quelque sorte et pour reprendre le terme de Husserl, des "consciences d'images ». Tout se passe, en fait, comme s'il existait dans le film une pensée mise en images qui, promulguant du temps et de l'espace, convoquerait le spectateur à la lecture - de ce que par ailleurs il est lui-même - d'une conscience-espace et d'une consciencetemps ou à celle, enfin, d'une sorte d'eidôlon: "[...] d'image qui reproduit la forme des objets [...] [et, le faisant, se manifeste]" (Jacob, p. 764). Un certain eidōlon bref qui, accomplissant du temps, laisse apparaître le visage d'un temps "perçu " et, par intrication, celui du temps "senti» de l'expérience humaine. 
$\mathrm{Si}$, de fait, le film a un caractère intentionnel, ce n'est qu'en vertu du fait qu'en décrivant des espaces et des temps, il laisse apparaître une certaine visée et, d'une autre façon sur le plan langagier, une connaissance prédicative. Or, on le voit, de cette manifestation éidétique de la connaissance - au sens husserlien d'intentionnalité - à l'effectuation de la découpe temporelle cognitive du film s'affiche une idée et un eidōlon, des images en forme de pensée ou, à l'inverse et pour marquer davantage le projet intentionnel du film, des pensées en formes d'images. Ces pensées peuvent paraître silencieuses, ces images peuvent bien être orientées vers la fiction, le projet intentionnel du film demeure.

Bref, sous le couvert d'une intentionnalité, ici filmique, et par ailleurs sous celui de l'ouvrage du film, deux temps sont, selon les termes husserliens, mis à la fois à contribution, et davantage, en intrication: le temps "perçu" et le temps "senti ${ }^{25}$ ". Le temps perçu est cette syntaxe, la part visible que donne à lire l'ouvrier temporel du film, alors que le temps senti est ce dépôt, cet écho, cette "nappe" sur laquelle repose et où retourne le temps perçu. C'est ce que voit le spectateur, à la fois la nappe et le dépôt, à la fois le temps de la perception et le temps en sa perception - qui comprend, intriqués, le "perçu " et le "senti», l'appréhendé - et par lequel, par recouvrement on l'a dit, le spectateur conçoit le temps, le film en tant qu'œuvre temporelle ${ }^{26}$.

Au cinéma cependant, pas de temps phénoménalement apparaissant du réel et de la quotidienneté, mais que des temps en manifestation d'apparition, en forme de pensée en image, de conscience-temps, de conscience perçue en travail de perception, laquelle impose, par syntaxe énoncée, une sorte de hiérarchie temporelle des vécus cognitifs entre temps "perçu» et temps "senti". Ici plus qu'ailleurs, et je rejoins là la pensée barthienne du langage phénoménologique de la photographie, "[...] on peut parler d'un "phénomène de l'être" dans la mesure même où il $\mathrm{y}$ a un "être du phénomène" [temporofilmique] (Dumont et Vandooren, p. 502); ici, plus qu'ailleurs, on peut établir une certaine correspondance entre le "punctum ou la déchirure du temps", l'intentionnalité filmique qui en fabrique les rives et les dérives et, enfin, cette conscience d'images, et de narrativité, mise en temps par le récit filmique. 
"Ce passage [dans l'"image» du film Shoah] du "était» au "ne plus" et le recouvrement de l'un par l'autre expriment seulement le double sens du présent [filmique], d'une part comme point-source, comme initiative d'une continuité rétentionnelle [posant le souvenir du temps passé par celui du quasi-présent écranique], et d'autre part comme point-limite, abstrait par division infinie du continuum temporel, [une impossible saisie du flux, du flou et du filé temporels](Ricœur, 1985, p. 61)».

Or, c'est cela précisément que le film Shoah donne à voir, un certain "punctum" temporel et mémoriel, l'ouvrage d'un produit intentionnel, filmique et, par lui, historique; celui du temps et de son effectuation ressouvenante en forme de nonperception directe - ou d'infiguration relative - et puis, cela, ce temps de l'expérience humaine et sa fuite en avant et en arrière dans la lecture "longitudinale" du film, mais aussi dans celle, transversale, que donnent à voir les écarts temporels compris dans les images mêmes, comme entre les voix et les images.

D'où il appert que le cinéma et, davantage, le film est à la fois ceci : un discours qui, de sa manifestation temporelle, agit comme un point-source, mais aussi phénoménalement, comme un point-limite. Au cinéma, pour reprendre un peu de l'exergue de cette dernière partie de cet essai, "nous n'avons affaire à la réalité que dans la mesure où elle est visée, représentée, intuitionnée, conceptuellement pensée", et nous n'avons affaire à la réalité que dans la mesure où le spectateur vise cette visée, ce passage de l'esprit du texte et de la conscience d'image à l'esprit de la réalité qu'elle conçoit. Ce travail est de part et d'autre, du côté de la pensée en écriture et de la pensée en lecture, perceptif et cognitif. On pourrait prétendre qu'il existe au cinéma une dissociabilité de la vitre et du paysage dans la mesure où œuvrent dans l'espace écranique, de façons longitudinale et transversale, la coupe du temps et le temps de son effectuation par la pensée qui persiste et dure.

Université Laval 
1 Je reprends dans le titre et l'exergue du présent texte, le terme en usage dans l'ouvrage La Chambre claire de Roland Barthes (Paris: Seuil, 1980, p. 15).

2 Gilles Deleuze, dans son ouvrage Cinéma 1. L'Image-mouvement (Paris: Minuit, 1983) précisait à propos de l'entreprise de cristallisation du film: "L'image-cristal a ces deux aspects: limite intérieure de tous les circuits relatifs, mais aussi enveloppe ultime, variable, déformable, aux confins du monde, au-delà même des mouvements du monde" (p. 108).

3 Sans faire allusion au temps contenu dans l'image, Roland Barthes parlait plus simplement, en page 18 de La Chambre claire, d'adhérence référentielle - et singulière. Lors d'une communication intitulée "L'enjeu d'une certaine hétérogénéité au cinéma " j'ai, en 1990, dans le cadre d'un colloque organisé par l'AQEC (Association québécoise des études cinématographiques), examiné cette question d'adhérence référentielle et, selon les termes de Schaeffer, celle du statut de l'empreinte et de l'imprégnant dans l'image et la syntaxe filmiques. Parce qu'il s'agit là de l'idée ici poursuivie, je cite cette communication : "[...] il importe, je crois, de considérer la présence au sein du système du renvoi au hors-champ du temps de la réalité par lequel, pour aller viter, le film se temporalise, et au hors-champ de l'espace par lequel il se fait empreinte de monde en retournant, même implicitement, le fait filmique à l'étude d'une sorte d'hétérogénéité qui ne relève pas ici de considérations sémiologiques, mais bien plutôt d'un examen qui, à la lumière de certaines inspirations phénoménologiques, peut contribuer à mettre en évidence certains autres enjeux de l'écriture filmique. [...] lorsque l'instantanéité s'introduit dans le film pour corrompre la continuité filmique, il s'ensuit des moments de profonde gravité ou, si l'on veut, de "densité " temporelle [...]. L'image photographique en nous montrant le voir, nous livre le vu [...] ». Cette réflexion sur la cinématographie - pour l'opposer à la photographie que le premier terme comprend - a par moi été reprise, remaniée et considérablement approfondie à l'aide notamment de certains concepts énoncés par Régis Durand (Le Regard pensif) et a été publié dans "Les réticences discursives à l'écran ou les voix négatives ", Marguerite Duras, Paris: Éditions Écriture, 1993).

4 Roland Barthes mentionnait, en page 17 de La Chambre claire: "La photographie appartient à cette classe d'objets feuilletés dont on ne peut séparer les deux feuillets sans les détruire: la vitre et le paysage [...]."

5 Voir cet appel à la notion de non-temps en citation dans la note 3.

6 Je reprends ici l'expression de Barthes utilisée en page 148 de l'ouvrage déjà cité.

7 Compensant le caractère fragmentaire de toute expérience dans le temps, Ricœur se demandait: "Ne faut-il pas introduire l'expérience d'un horizon temporel [...]?" (Temps et récit. Le Temps raconté, Paris: Seuil, 1985, p. 90). J'ajoute, parce que la citation suivante contribuera sans doute à éclairer le propos "cognitif" et "temporel " et, par ailleurs, l'emploi du pluriel des horizons compris dans l'intitulé du présent article que: «L'expression d'horizon de vécu ne désigne pas seulement [...] l'horizon de temporalité phénoménologique. [...], mais des différences introduites par des modes de donnés répondant à un nouveau type. En ce sens un vécu qui est devenu un objet pour un regard du moi et qui a par conséquent le mode du regardé, a pour horizon un arrière-plan d'inattention qui présente des différences relatives de clarté et d'obscurité, ainsi que de relief et d'absence de relief " (Husserl, Edmond, Idées directrices pour une phénoménologie, Paris: Gallimard, 1985, p. 277-280 - la traduction est de P. Ricœur).

8 Bien que cette partie de la phrase ait été élidée, il faut savoir que Barthes fait mention de d'eidōlon émis par l'objet. J'en reparle plus loin.

9 "Le punctum d'une photo, disait Barthes, c'est ce hasard qui, en elle, me point (mais aussi me meurtrit, me poigne)" (La Chambre claire, Paris: Seuil, 1980, p. 49). 
10 Je fais référence à l'ouvrage de Paul Ricœur, La Métaphore vive, dans lequel était abordée la question de l'énonciation métaphorique. L'auteur avançait l'idée que la métaphore ne pouvait émerger de la seule présence d'un mot dans la phrase, mais était, au contraire, mise en évidence par le champ de l'énoncé ou celui de l'énonciation métaphorique qui la produit.

11 Roland Barthes disait: "Je ne puis que la balayer du regard, comme une surface étale" (La Chambre claire, Paris: Seuil, 1980, p. 164).

12 "Il n'y a pas de doute: le problème est [ici, comme ailleurs] celui de la durée comme même. Et la rétention, simplement nommée ici, est le nom de la solution cherchée" (Temps et récit 3. Le Temps raconté, Paris: Seuil, 1985, p. 51).

13 Voir à propos notamment d'une exploration de la notion d' "intervalle " au cinéma, le fameux ouvrage de Marie-Claire Ropars, Le Texte divisé. Je fais cependant, et pour l'ensemble du présent texte, référence au troisième tome de l'ouvrage de Paul Ricœur, Temps et récit, 3. Le Temps raconté (Paris: Seuil, 1985) et, plus particulièrement, au chapitre "Temps intuitif ou temps invisible? Husserl face à Kant " en pages 43 à 109.

14 Je privilégierai dorénavant le plus souvent, pour éviter toute confusion de position théorique, le terme de pensée en écriture plutôt que, comme je l'ai souvent fait, d'employer le terme de scriptural - et son dérivé le scripteur — privilégié par Ropars dans, notamment, Le Texte divisé. Notons que si l'hypothèse qu'appelle l'emploi de ce terme m'a en quelque sorte servi de sol à la conduite d'une étude d'inspiration sémiologique, psychanalytique et, davantage, phénoménologique qui portait de façon frontale sur les silences au cinéma, la trajectoire que j'empruntais concernait, elle, en bout de course, celle de l'identité en écriture. La lecture des ouvrages de Marie-Claire Ropars est, à l'égard de l'écriture filmique, et de son hypothèse scripturale, d'un très riche enseignement.

15 "Ả tout "noème" (contenu visé) correspond une certaine "noèse", un certain type d'intentionnalité (la "noèse" est l'acte de connaissance, le "noème" est le contenu relatif à cet acte, la connaissance en tant que résultat)" (Bruxelles: Les Dictionnaires Marabout Université Savoir Moderne, tome III, p. 501).

16 L'identité des vécus cognitifs est hiérarchisée par l'ensemble des vécus cognitifs relatifs à la réalité que l'écriture du film convoque toujours.

17 Les citations précédentes et suivantes sont tirées du film Shoah de Lanzmann.

18 Roland Barthes, toujours dans La Chambre claire (Paris: Seuil, 1980), disait: "L'important c'est que la photo possède une force constative, et que le constatif de la Photographie porte, non sur l'objet, mais sur le temps. D'un point de vue phénoménologique, dans la Photographie, le pouvoir d'authentification prime le pouvoir de représentation" (p. 138-139). Je voudrais convier le lecteur à consulter l'article de Marie-France Osterero "De l'histoire à la mémoire dans Shoah", Hors Cadre, $\mathrm{n}^{\circ} 9$ (1991). L'auteure y avance l'idée que sa lecture du film va dans le même sens que la mienne: "La parole en acte par son pouvoir d'évocation d'un lieu et d'un moment singulier n'explique pas la trace comme effet-signe du passé, elle nous la fait voir. Elle abolit pour un moment la distance du présent au passé, ou plutôt fait surgir le passé virtuellement présent dans l'image. Le lieu de la mémoire coïncide avec le lieu actuel présent sous nos yeux, sans pourtant s'y confondre: nous savons que nous ne voyons pas» (p. 99).

19 Voir à ce propos, en page 140 de l'ouvrage La Chambre claire (Paris: Seuil, 1980), la différence que fait Barthes entre le cinéma et la photographie.

20 Ricœur, dans ce même chapitre de Temps et récit 3. Le Temps raconté (Paris: Seuil, 1985) traitant de la pensée husserlienne, disait ceci : "Ce "re" est alors décrit comme un phénomène de "correspondance" terme à terme, dans lequel, par hypo- 
thèse, la différence n'est pas de contenu [...], mais de mode d'accomplissement " (p. 62).

21 Je cite, en simplifiant, " [...] la re-présentation est à sa façon une impression et une impression présente" (Temps et récit 3. Le Temps raconté, Paris: Seuil, 1985, p. 81).

22 Les Heidegger, Husserl, Derrida, Ropars, etc. se sont attachés à l'étude de la ou des mémoire-s ou, pour le dire autrement, à celle de la charge mémorielle de l'écriture.

23 Le terme "conscience d'image" est de Husserl et tiré de son ouvrage Chose et espace. Leçons 1907 (Paris: P.U.F., 1989, p. 11) - je reprends le terme sans nécessairement convoquer les idées qu'il comprend.

24 On sait combien le projet phénoménologique de Husserl avait pour objectif et pour méthode la description des phénomènes. "[...] ce qui nous intéresse, ce sont les vécus cognitifs d'après leur sens objectif et leur teneur descriptive" (Ricœur, 1985, p. 15). Je rejoins là, pour ce qui concerne la présente trajectoire d'analyse du langage cinématographique, certains énoncés compris dans la conclusion de l'article "Langage cinématographique et faillibilité" paru dans Cinémas, vol. 4, no 3 (1994). L'être-là dont il était question correspondait alors à une couche de la pensée, un là, une pensée sans intentionnalité avouée, un là qui connaît. L'intentionnalité reçoit l'acception, descriptive, d'une activité cognitive, d'un faire-pensée. "L'orientation-vers, disait Husserl, est elle-même un "je fais", et de même le va-et-vient des rayons du regard attentif, du regard dans le mode de l'orientation-vers, est un "je fais" " (Expérience et jugement, Paris: P.U.F., 1970, p. 100). Notons par ailleurs que, dans une autre perspective, Edmond Couchot fait référence au faire filmique dans son article "Audelà du cinéma. Images et temps numériques".

25 Je me permets de citer ici assez longuement Husserl qui, mieux que je ne saurais le faire, explique cet écart relatif entre le temps "senti » et le temps "perçu " qu'il conçoit. Je parle pour ce qui me concerne de leur intrication et je n'exclus pas la connaissance d'un horizon temporel, par intentionnalité et cognitivité, pluriel - voir à ce propos le titre du présent article et la citation comprise dans la note 7 qui en motive en quelque sorte les inspirations. Le temps "senti " s'oppose au deuxième (au temps perçu) " [...] par l'aperception empirique duquel se constitue la relation au temps objectif. [...] Le temps objectif appartient à l'enchaînement de l'objectivité de l'expérience. Les data de temps "sentis" ne sont pas simplement sentis, ils sont aussi chargés de caractères d'appréhension, et à ces derniers appartiennent à leur tour certaines exigences et certaines possibilités légitimes : la possibilité de mesurer les uns aux autres les temps et les relations de temps qui, sur la base de temps "sentis", apparaissent; celle de les placer de telle et telle manière dans des ordres objectifs, celle de les séparer de telle et telle manière en ordres apparents et réels. Ce qui se constitue là comme être objectivement valable est finalement l'unique temps objectif infini, dans lequel toute chose et tout événement [...], les âmes avec leurs états psychiques, ont leur place temporelle déterminée, déterminable par le chronomètre " (Leçons pour une phénoménologie de la conscience intime du temps, Paris: P.U.F., 1964, p. 12).

J'exclus du cadre de cette étude l'examen des ambiguïtés qu'a relevées Ricœur et qui, selon ses termes, ont trait à la prétention des analyses phénoménologiques de " [...] s'affranchir de toute référence à un temps objectif et à atteindre par réflexion directe une temporalité purifiée de toute visée transcendante" (Temps et récit 3. Le Temps raconté, Paris: Seuil, 1985, p. 82). Avec toutes les précautions qu'impose l'actuel projet, qui examine le cinéma en s'inspirant de certaines données phénoménologiques plutôt que de traiter des questions proprement phénoménologiques elles-mêmes et pour justifier un peu - mais autrement, au regard d'une phénoménologie du cinéma - le projet d'étude proposé par Husserl, je souligne le fait que sa pensée théorique ne tient pas uniquement à une réflexion directe du temps, mais précisément de 
la conscience intime du temps. Il tend aussi, en bout de course, à une pratique descriptive de l'indescriptible du temps, seulement " senti ", à l'impossible «identité" par la pensée du flux temporel et, autrement, aux termes d'une phénoménologie langagière, à une sorte de hiérarchie des vécus cognitifs temporels. Sans donc se livrer à l'examen du questionnement phénoménologique du temps, je peux prétendre que les propos de la perception, de l'intentionnalité, de la rétention et du ressouvenir fournissent un site où réfléchir sur la pensée en écriture dans le film.

Bref, et c'est cet examen qui nous intéresse, l'écriture filmique matérialise cette intentionnalité temporelle dans un matériau filmique qui est aussi un matériau de perception et de cognition et où se manifeste une certaine hiérarchie des vécus cognitifs, des " reliefs " temporels et "l'absence — appréhendée — de reliefs" temporels.

26 Là est, je le souligne encore, le propos de Ricœur dans les trois tomes de Temps et récit.

\section{OUVRAGES CITÉS}

Barthes, Roland. La Chambre claire. Paris: Seuil, 1980.

Deleuze, Gilles. Cinéma 1. L'Image-mouvement. Paris: Minuit, 1983.

Dumont, Jacques et Vandooren, P. (direction). La Philosophie. Bruxelles: Les Dictionnaires Marabout Université Savoir moderne, tome III, 1972.

Husserl, Edmond. Chose et espace. Leçons 1907. Paris: P.U.F., 1989.

Husserl, Edmond. Expérience et jugement. Paris: P.U.F., 1970a.

Husserl, Edmond. Idées directrices pour une phénoménologie. Paris: Gallimard, 1985.

Husserl, Edmond. L'Idée de la phénoménologie. Paris: P.U.F., $1970 \mathrm{~b}$.

Husserl, Edmond. Leçons pour une phénoménologie de la conscience intime du temps. Paris : P.U.F., 1964.

Jacob, André. Les Notions philosophiques. Dictionnaire. Paris: P.U.F., 1990.

Ricœur, Paul. La Métaphore vive. Paris: Seuil, 1975.

Ricœur, Paul. Temps et récit 3. Le temps raconté. Paris: Seuil, 1985.

Roy, Lucie. Le Silence au cinéma ou les structures de l'inexistant II. Thèse de doctorat. Québec:Université Laval, 1989.

\section{FILMOGRAPHIE}

Lanzmann, Claude. Shoah. France: Les Films Aleph, 1985. 